\title{
Road Quality Information Based Adaptive Semi-active Suspension Control
}

\author{
Hakan Basargan*, András Mihály², Péter Gáspár², Olivier Sename³ \\ 1 Department of Control for Transportation and Vehicle Systems, Faculty of Transportation and Vehicle Engineering, \\ Budapest University of Technology and Economics, H-1111 Budapest, 2 Stoczek street, Hungary \\ 2 Systems and Control Laboratory, Institute for Computer Science and Control (SZTAKI), Eötvös Loránd Research Network (ELKH), \\ H-1111 Budapest, 13-17 Kende street, Budapest, Hungary \\ ${ }^{3}$ GIPSA-lab, INPG, Université Grenoble Alpes, 38402 Grenoble, 11 Rue des Mathématiques, France \\ * Corresponding author, e-mail: hakan.basargan@kjk.bme.hu
}

Received: 18 May 2021, Accepted: 15 June 2021, Published online: 16 July 2021

\begin{abstract}
This paper introduces an adaptive semi-active suspension control by considering global positioning system-based and historical road information. The main idea of this study is to find a corresponding trade-off between comfort and stability at different road irregularities. The introduced semi-active controller is designed based on the Linear Parameter-Varying framework. The behavior of the designed controller can be modified by the use of a scheduling variable. This scheduling variable is selected by considering the various road category. TruckSim simulation environment is used in order to validate the introduced adaptive semi-active suspension control system by comparing it with the non-adaptive scenario. The results show that both driving comfort and vehicle stability have been improved with the proposed adaptive semi-active suspension control.
\end{abstract}

Keywords

semi-active suspension control, adaptive semi-active suspension control, LPV control, ride comfort, vehicle stability

\section{Introduction}

The vehicle suspension system is one of the key topics in vehicle technology due to its important role in vehicle safety, stability, ride comfort and overall performance of the vehicle. The vehicle suspension system consists of the spring, wishbones and the shock observer. The damper aims to damp body and wheel oscillations, thus it contributes to both driving comfort and safety. The spring carries the body-mass for isolating the vehicle body from road distortions. Suspension systems are classified as active, passive, semi-active and several in-between systems.

The suspension system needs to satisfy several important requirements such as guaranteeing vehicle safety, road holding and driving comfort, avoiding extreme suspension stroke, maintaining proper vehicle condition subject to external and internal forces (Tsend and Hrovat, 2015). It is difficult to adjust the spring's stiffness, thus many of the semi-active suspension systems are realized by changing the damping. Therefore, the most important actuator is a variable-damping damper in the semi-active suspension systems. These dampers are classified into electrorheological, magnetorheological and solenoid valve types (Gong and Chen, 2020). In this study, magnetorheological(MR) damper type is modeled.

There are several control approaches for the semi-active suspension control. The skyhook control strategy has been commonly used in semi-active suspension control (Liu et al., 2019; Moaaz and Ghazaly, 2019; Shimoya and Katsuyama, 2019). This strategy is based on designing the active suspension control so that the chassis is linked to the sky to reduce the vertical accelerations of the chassis and of the axle independently of each other. It is easy to implement with few state information and effective in order to enhance the vehicle's ride comfort, however it disrupts the dynamic tire load. The model predictive control (MPC) (Madhavan Rathai et al., 2019a; Madhavan Rathai et al., 2019b) and hybrid model predictive control (Hybrid MPC) (Zhang et al., 2017) methods are also commonly used but they still lack robustness properties and their application is not easy. Unlike previous methods, $\boldsymbol{H}_{\infty}$ control approach (Ye and Zheng, 2019; Yu and Zhang, 2019) guarantees road holding, 
vehicle stability, and ride comfort performances, however dynamic control reconfiguration is not possible due to the fixed weighting of the performances. Control reconfiguration has key importance in the control of the semi-active suspension systems in order to configure the controller according to different road conditions. For instance, the damper needs to act differently for each road irregularities, such as bumps, potholes, roughness, etc. Thus, the semi-active suspension control is founded on the Linear ParameterVarying (LPV) framework in this study due to its availability for the dynamic configuration by modifying the scheduling variable, see (Basargan et al., 2020a; Basargan et al., 2020b; Basargan et al., 2021; Morato et al., 2019; Pham, 2020). LPV control method describes an approach for the semi-active suspension controller with consideration of actuator constraints. This method offers a flexible framework for the designing of the nonlinear controllers. Another reason to use this method in our study is its flexibility.

The paper is organized as follows: Section 2 presents the quarter-car model, which is control-oriented, and the LPV control synthesis. Section 3 introduces the integration of the system and the method of defining the scheduling variable based on GPS-based road data. Validation of the introduced method has been done in Section 4 in TruckSim simulation environment. Finally, conclusions are drawn in Section 5.

\section{Modeling and control design of the system}

This section contains modeling of the quarter-car suspension and designing of the reconfigurable semi-active suspension control through LPV method.

\subsection{Quarter-car suspension model}

The quarter-car model, which is the most employed and useful is shown in Fig. 1. This model is used due to its simplicity and controllability, while it has 2 degrees-of-freedom.

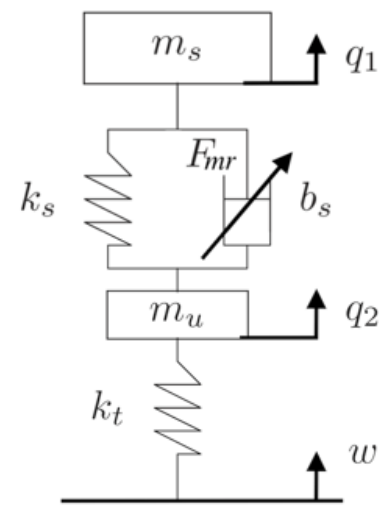

Fig. 1 Quarter-car model
The two degrees of freedom are sprung mass and the unsprung mass displacements. The tire and suspension systems are denoted by a damper and spring. The dynamics of the quarter-car semi-active suspension system and the actuator is written in Eq. (1) and (2).

$$
\begin{aligned}
& m_{s} \ddot{q}_{1}+b_{s}\left(\dot{q}_{1}-\dot{q}_{2}\right)+k_{s}\left(q_{1}-q_{2}\right)+F_{m r}=0 ; \\
& m_{u} \ddot{q}_{2}+b_{s}\left(\dot{q}_{2}-\dot{q}_{1}\right)+k_{t}\left(q_{2}-w\right) \\
& +k_{s}\left(q_{2}-q_{1}\right)-F_{m r}=0 . \\
& \dot{F}_{m r}=-\frac{1}{\tau} F_{m r}+\frac{1}{\tau} u .
\end{aligned}
$$

Here, $m_{s}$ and $m_{u}$ denote the sprung and unsprung masses of the quarter-car, $k_{t}$ and $k_{s}$ are stiffness of the tire and spring, $b_{s}$ is the damping rate of the shock absorber, $F_{m r}$ is the control force generated by the actuator and $u=F_{m r}$ is the control input of the system. Vertical displacement of the sprung and unsprung mass is denoted with $q_{1}$ and $q_{2}$, while road disturbance is denoted with $w$. Parameters of the quarter-car model for both rear and front suspension are shown in the Table 1.

\subsection{Control synthesis}

In the design process, state vector $\boldsymbol{x}$ is selected as $\boldsymbol{x}=\left[x_{1} x_{2} x_{3} x_{4} x_{5}\right]^{T}$, where components are shown in Eq. (3).

$x_{1}=q_{1}$

$x_{2}=q_{2}$

$x_{3}=\dot{q}_{1}$

$x_{4}=\dot{q}_{2}$

$x_{5}=F_{m r}$,

where, $q_{1}$ and $q_{2}$ are vertical displacement of sprung and unsprung masses, $\dot{q}_{1}$ and $\dot{q}_{2}$ are vertical velocity of sprung and unsprung masses. The unmodeled dynamics $\Delta$ are taken into consideration with $\left|\Delta\left(w_{1}\right)\right|=25$ at low and $\left|\Delta\left(w_{2}\right)\right|=1$ at high frequencies while it is assumed to be stable with the norm condition $\|\Delta\|_{\infty}<1$.

However, performance specifications need to be defined in order to achieve a desirable trade-off between road holding and ride comfort and control force also needs

Table 1 Parameters of the suspension

\begin{tabular}{lccc}
\hline Parameters(symbols) & $\begin{array}{c}\text { Front } \\
\text { suspension }\end{array}$ & $\begin{array}{c}\text { Rear } \\
\text { suspension }\end{array}$ & Unit \\
\hline Suspension stiffness $\left(k_{s}\right)$ & 30 & 60 & $\mathrm{kN} / \mathrm{m}$ \\
Tire stiffness $\left(k_{t}\right)$ & 220 & 220 & $\mathrm{kN} / \mathrm{m}$ \\
Sprung mass $\left(m_{s}\right)$ & 214 & 336 & $\mathrm{~kg}$ \\
Unsprung mass $\left(m_{u}\right)$ & 40 & 40 & $\mathrm{~kg}$ \\
Time constant $(\tau)$ & $1 / 30$ & $1 / 30$ & $\mathrm{~s}$ \\
Damping $\left(b_{s}\right)$ & 50 & 50 & $\mathrm{~N} / \mathrm{m} / \mathrm{s}$ \\
\hline
\end{tabular}


to be considered. Body acceleration must be minimized to increase passenger comfort with $z_{1}$ criterion. At the same time, the stability of the vehicle is guaranteed with suspension deflection minimization with $z_{2}$. The dynamic tire load has to be minimized to decrease variations of slide force to provide a guarantee for the stability with $z_{3}$ and the control force must be considered with $z_{4}$, These optimization criteria can be found in Eq. (4).

$$
\begin{aligned}
& z_{1}=\dot{q}_{1} \rightarrow 0 \\
& z_{2}=\left(q_{1}-q_{2}\right) \rightarrow 0 \\
& z_{3}=\left(q_{2}-w\right) \rightarrow 0 \\
& z_{4}=F_{m r} \rightarrow 0 .
\end{aligned}
$$

Finally, these performances are inserted in the performance vector, $\mathbf{z}=\left[z_{1} z_{2} z_{3} z_{4}\right]$.

Then, the system is given with Eq. (1) needs to be transformed into the state-space representation form as Eq. (5).

$$
\begin{aligned}
& \dot{\mathbf{x}}=A_{x}+B_{1} w+B_{2} u \\
& \mathbf{z}=C_{1} x+D_{11} w+D_{12} u \\
& \mathbf{y}=C_{2} x+D_{21} w+D_{22} u .
\end{aligned}
$$

Matrices are found below:

$$
\begin{aligned}
& A=\left[\begin{array}{ccccc}
0 & 0 & 1 & 0 & 0 \\
0 & 0 & 0 & 1 & 0 \\
-\frac{k_{s}}{m_{s}} & \frac{k_{s}}{m_{s}} & -\frac{b_{s}}{m_{s}} & \frac{b_{s}}{m_{s}} & -\frac{1}{m_{s}} \\
\frac{k_{s}}{m_{u}} & \frac{-\left(k_{s}+k_{t}\right)}{m_{u}} & \frac{b_{s}}{m_{u}} & -\frac{b_{s}}{m_{u}} & \frac{1}{m_{u}} \\
0 & 0 & 0 & 0 & -\frac{1}{\tau}
\end{array}\right], \\
& B_{1}=\left[\begin{array}{lllll}
0 & 0 & 0 & \frac{k_{t}}{m_{u}} & 0
\end{array}\right]^{T}, \\
& B_{2}=\left[\begin{array}{lllll}
0 & 0 & 0 & 0 & \frac{1}{\tau}
\end{array}\right]^{T}, \\
& B=\left[\begin{array}{ll}
B_{1} & B_{2}
\end{array}\right], \\
& C_{1}=\left[\begin{array}{ccccc}
-\frac{k_{s}}{m_{s}} & \frac{k_{s}}{m_{s}} & -\frac{b_{s}}{m_{s}} & \frac{b_{s}}{m_{s}} & -\frac{1}{m_{s}} \\
1 & -1 & 0 & 0 & 0 \\
0 & 1 & 0 & 0 & 0 \\
0 & 0 & 0 & 0 & 1
\end{array}\right] \text {, } \\
& C_{2}=\left[\begin{array}{lllll}
1 & -1 & 0 & 0 & 0
\end{array}\right], \\
& C=\left[\begin{array}{l}
C_{1} \\
C_{2}
\end{array}\right],
\end{aligned}
$$

$$
\begin{aligned}
& D_{11}=\left[\begin{array}{llll}
0 & 0 & -1 & 0
\end{array}\right]^{T}, \\
& D_{12}=\left[\begin{array}{llll}
0 & 0 & 0 & 0
\end{array}\right]^{T}, \\
& D_{21}=[0], \\
& D_{22}=\left[\begin{array}{ll}
0
\end{array}\right], \\
& D=\left[\begin{array}{ll}
D_{11} & D_{12} \\
D_{21} & D_{22}
\end{array}\right] .
\end{aligned}
$$

The introduced controller is founded on a weighting strategy formulated with a closed-loop architecture, see Fig. 2. $G$ is the quarter-car model, defined in Eq. (5), $K$ is the designed LPV controller with scheduling variable $\rho, y$ is the measured output, $u$ is the control input, $z$ denotes the performance outputs, $n$ expresses the measurement noise and $w$ is the road disturbance.

The weighting functions $W_{w}$ and $W_{n}$ represent road disturbances and sensor noise. The goal of $W_{p}$ is to keep the sprung mass acceleration $\left(W_{p a}\right)$, suspension deflection $\left(W_{p d}\right)$, tire deformation $\left(W_{p t}\right)$ and control input $\left(W_{p u}\right)$ small over the required frequency range. The weighting functions $W_{p t}$ and $W_{p d}$ are for the tire load and stability while $W_{p a}$ stands for the driving comfort. It is necessary to define scheduling variable $\rho \in[0,1]$ to shape the weighting functions ( $W_{p a}, W_{p t}$ and $W_{p d}$ ) to guarantee the control configuration in case of the predefined performances become more significant by the reason of estimated oncoming road conditions. These weighting functions are in a second-order proportional form and they are found in Eq. (6). The weighting functions $W_{p u}, W_{r}, W_{n}$ and $W_{w}$ are all given in similar proportional and linear form without containing the scheduling variable.

$$
\begin{aligned}
& W_{p t}=(1-\rho) \frac{\alpha_{3} s+1}{T_{3} s+1} \\
& W_{p d}=(1-\rho) \frac{\alpha_{2} s+1}{T_{2} s+1} \\
& W_{p a}=\rho \frac{\alpha_{1} s+1}{T_{1} s+1},
\end{aligned}
$$

where, $T_{1.2 .3}$ and $\alpha_{1.2 .3}$ are design parameters. The Bode diagram of the weighting functions is shown in Fig. 3.

The LPV technique ensures a rigorous framework for designing the nonlinear controllers for time-varying plants. These plants are characterized by accurate assumption on the exogenous scheduling variables, such as rate of variations, bounds on the magnitudes and the availability of an estimated or measured value of the current scheduling variable during operation (Flep et al., 2016). The LPV 


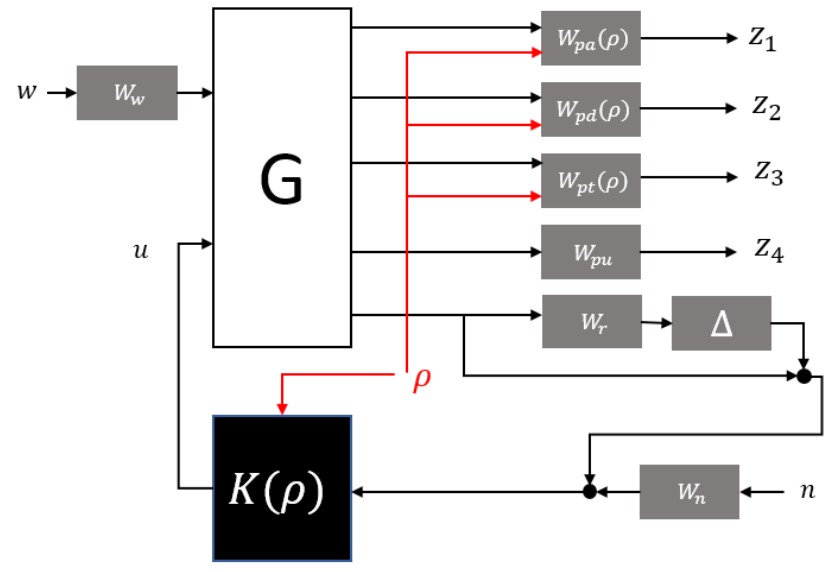

Fig. 2 Closed-loop interconnection

performance problem is to choose a parameter varying control, which guarantees quadratic stability for the closed-loop system while the induced $L_{2}$ from the disturbance $w$ to the performances $z$ is smaller than the value $\gamma$, as described in (Bokor and Balas, 2005). Hence, the minimization task is given in Eq. (7).

$$
\begin{aligned}
& \inf \sup \quad \text { sup } \\
& K \varrho \in F_{p} w_{2} \neq 0, w \in \mathcal{L}_{2} \frac{z_{2}}{w_{2}} \leq \gamma .
\end{aligned}
$$

The solution of the problem is governed by the set of infinite dimensional LMIs being satisfied for all $\rho \in F_{p}$, thus it is a convex problem. In practice, this problem is set up by gridding a parameter space and solving the set of the LMIs that hold on the subset of $F_{p}$, see (Wu et al., 1995).

The introduced design results in a reconfigurable LPV controller, where $\rho=1$ stands for the performance of driving comfort and $\rho=0$ stands for road holding and vehicle stability, while between these edge values a mixed performance is given.

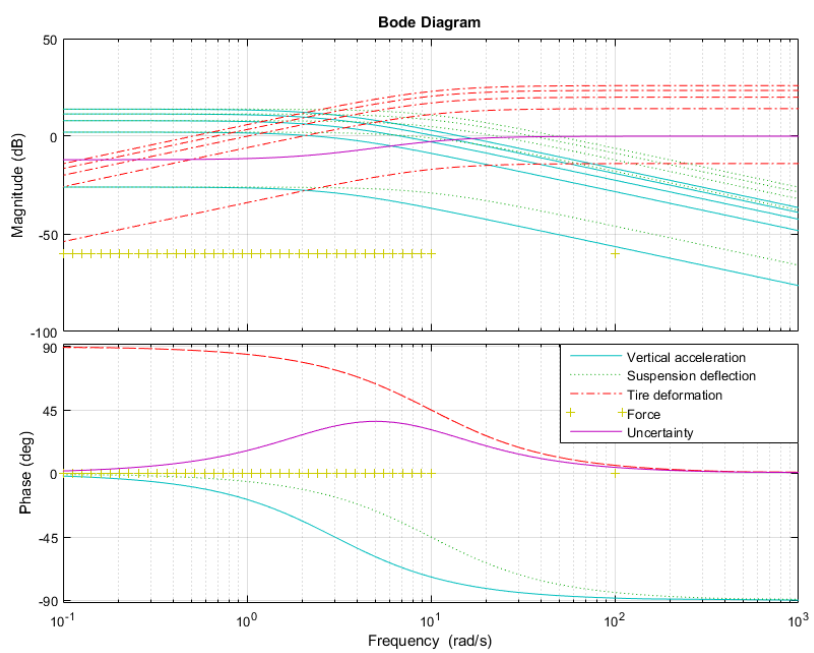

Fig. 3 Weighting functions
The MR damper has a limitation on the control input force, thus a semi-optimal solution is given in Eq. (8).

$F_{m r}= \begin{cases}F_{\text {opt }}, & \text { if } \dot{q}_{1}\left(\dot{q}_{1}-\dot{q}_{2}\right)>0 \\ F_{p a s}, & \text { if } \dot{q}_{1}\left(\dot{q}_{1}-\dot{q}_{2}\right)<0\end{cases}$

Here, $F_{o p t}$ and $F_{p a s}$ are optimal and passive suspension force and $\dot{q}_{1}-\dot{q}_{2}$ is the deflection velocity of the suspension.

\section{System integration and decision layer}

System integration and decision layer, which selects the suitable scheduling variable for the controller will be explained in this section.

Integration of the system is shown in Fig. 4. Measured output $y=q_{1}-q_{2}$ is relative displacement between masses, while control input for the system is the vertical force generated by the damper. GPS based road data consists of GPS points and location of the road distortions. It is assumed that the location of road irregularities is known according to previous measurements. Decision later selects the compatible scheduling variable based on the GPS based road data and previous measurements.

Real time road information, such as location of bumps or irregularities has a really significant role in the suspension system. Thus, controlled suspension system should be adapted to the road conditions and response time for road irregularities must be fast. First of all, categorization of road condition is necessary to detect the effect of the oncoming road irregularities. The ISO 2631-1:1997 standard (International Organization for Standardization, 1997) is considered to categorize the road distortions and irregularities. This standard characterizes the ride comfort based on the action of the vibration on the drivers and passengers. The used standard can be seen in Table 2. The frequency-weighted vibration magnitude (FWVM) is calculated as

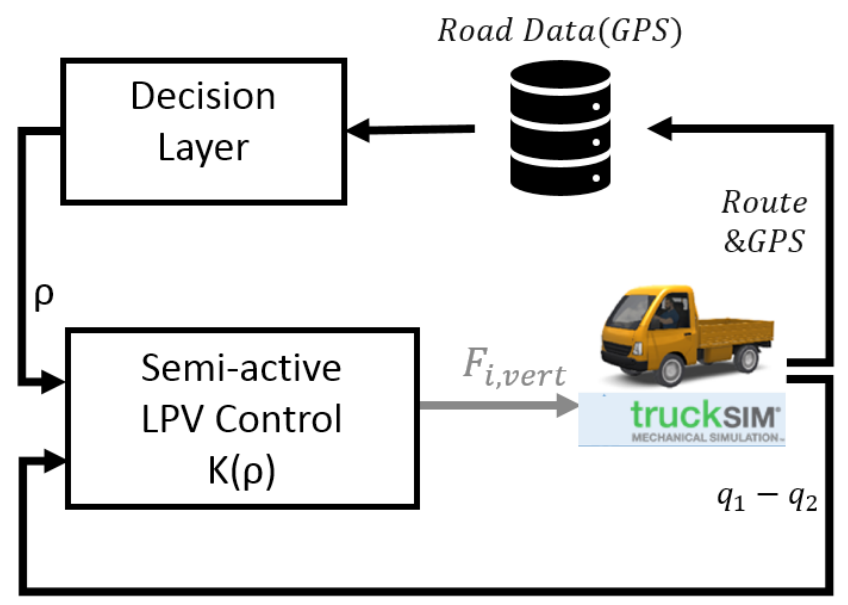

Fig. 4 System integration 
the root mean square (RMS) value of the vertical acceleration of the body. Equation (9) provides the solution of the RMS calculation, where $a(t)$ is the time-weighted acceleration and $T$ is the number of the acceleration values.

$a_{R M S}=\sqrt{\frac{1}{T} \int_{0}^{T} a^{2}(t) d t}$

Many passive suspension simulations have been done to categorize the simulated road irregularities. Vertical acceleration, tire deformation and suspension deflection results of the passive suspension simulations in different velocity and road irregularities are analyzed and vertical acceleration results have been used for the ISO standard (International Organization for Standardization, 1997). An example is shown in Table 3 with a vehicle velocity of $20 \mathrm{~km} / \mathrm{h}$. According to these results, categorized road types and their FWVM values are listed in Table 3.

Here, the roughness road and $10 \mathrm{~cm}$ bump road types are in the "Not uncomfortable" road category with 0.026 and $0.116 \mathrm{~m} / \mathrm{s}^{2} \mathrm{FWVM}$ value respectively, while several bumps and sine-sweep road types are in the "A little uncomfortable" road category with 0.37 and $0.431 \mathrm{~m} / \mathrm{s}^{2} \mathrm{FWVM}$ value respectively. These road irregularities are seen in Fig. 5.

The FWVM value of the "Not uncomfortable" road category is less than $0.315 \mathrm{~m} / \mathrm{s}^{2}$, thus there is no comfort-related issue. Herewith, mainly stability and road holding performances are prioritized by selecting the scheduling variable as $\rho=0.25$. However, the FWVM value of the "A little uncomfortable" road category is between 0.315 and $0.63 \mathrm{~m} / \mathrm{s}^{2}$, comfort-related, stability-related and road holding-related performances are evaluated identically to make the importance of these performances equal. This equal importance is selected by choosing scheduling

Table 2 Magnitudes of overall vibration (ISO 2631-1:1997)

\begin{tabular}{lc}
\hline FWVM & Comfort category \\
\hline Less than $0.315 \mathrm{~m} / \mathrm{s}^{2}$ & Not uncomfortable \\
$0.315-0.63 \mathrm{~m} / \mathrm{s}^{2}$ & A little uncomfortable \\
$0.5-1 \mathrm{~m} / \mathrm{s}^{2}$ & Fairly uncomfortable \\
$0.8-1.6 \mathrm{~m} / \mathrm{s}^{2}$ & Uncomfortable \\
$1.25-2.5 \mathrm{~m} / \mathrm{s}^{2}$ & Very uncomfortable \\
Greater than $2 \mathrm{~m} / \mathrm{s}^{2}$ & Extremely uncomfortable \\
\hline
\end{tabular}

Table 3 Road irregularities

\begin{tabular}{lcc}
\hline Road type & FWVM $\left(\mathrm{m} / \mathrm{s}^{2}\right)$ & Road category \\
\hline Rough road / Road roughness & 0.026 & Not uncomfortable \\
$10 \mathrm{~cm}$ bump & 0.116 & Not uncomfortable \\
Several bumps & 0.370 & A little uncomfortable \\
Sine sweep & 0.431 & A little uncomfortable \\
\hline
\end{tabular}
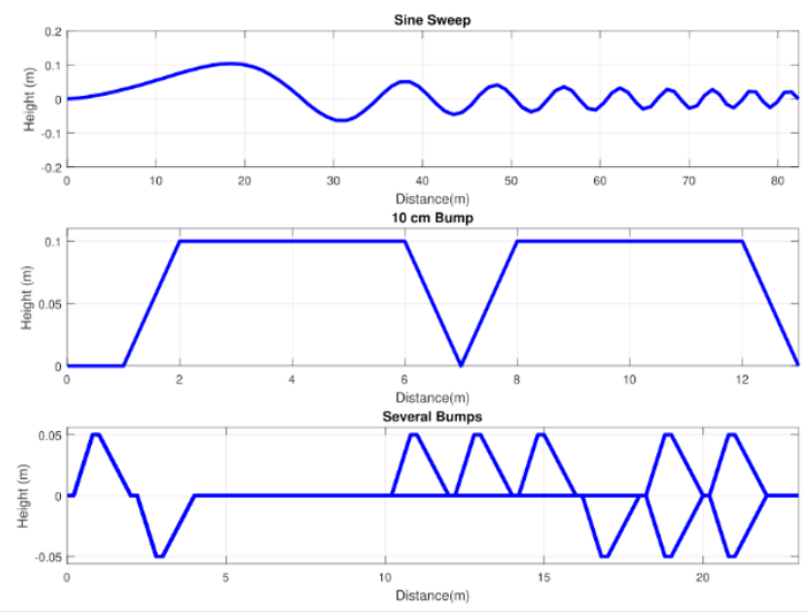

Fig. 5 Road irregularities

variable as $\rho=0.5$. The scheduling variable for the flat road is selected as $\rho=0$ since there is no comfort-related problem, while vehicle stability is guaranteed in curvatures and uphill/downhill roads.

\section{Simulation results}

The simulation has been run in TruckSim environment with a real geographical highway route. The effectiveness of the introduced method is demonstrated with two different simulations, which are the utility truck having adaptive semi-active suspension control and conventional semi-active suspension. The simulations have been run with constant velocity $(70 \mathrm{~km} / \mathrm{h})$. Table 4 shows the parameters of the simulation vehicle. Note, that the simulation vehicle has a $500 \mathrm{~kg}$ payload mass.

As it is shown in Fig. 6, the simulation route has $1700 \mathrm{~m}$ length with 3 different road irregularities in 4 locations. The simulation route has 3 curvatures, which are located at $400 \mathrm{~m}, 1000 \mathrm{~m}$, and $1600 \mathrm{~m}$. The selection of the road irregularities is based on real road scenarios. The $100 \mathrm{~mm}$ heightened right and left side bumps represent the speed bumps. These speed bumps are common in urban areas and it is possible to analyze driving over a speed bump with that irregularity. The several bumps with potholes and bumps following each other differently on the right

Table 4 Parameters of the simulation vehicle

\begin{tabular}{lcc}
\hline Parameter & Value & Unit \\
\hline Truck mass $\left(m_{t}\right)$ & 760 & $\mathrm{Kg}$ \\
Payload mass $\left(m_{p}\right)$ & 500 & $\mathrm{Kg}$ \\
Distance from C.G to front axle $\left(l_{1}\right)$ & 0.55 & $\mathrm{~m}$ \\
Distance from C.G to rear axle $\left(l_{2}\right)$ & 1.375 & $\mathrm{~m}$ \\
Track width $(b)$ & 500 & $\mathrm{Kg}$ \\
Height of COG $\left(h_{C O G}\right)$ & 0.55 & $\mathrm{~m}$ \\
Maximal suspension deflection $\left(d_{\max }\right)$ & 1.375 & $\mathrm{~m}$ \\
\hline
\end{tabular}



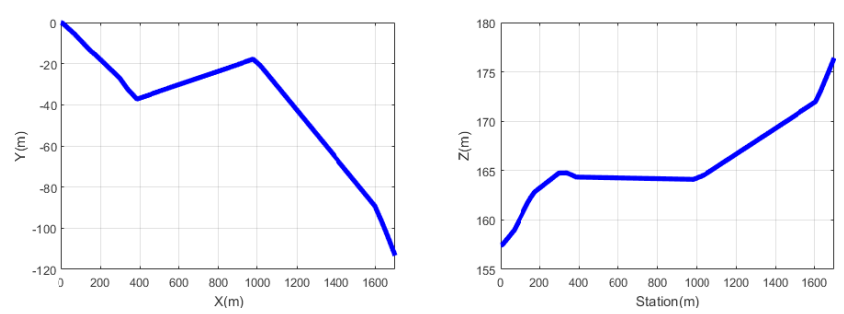

Fig. 6 Geometry of the selected road

and left side of the lane, having a depth and height of $50 \mathrm{~mm}$ represent bad road quality with discontinuities in the asphalt. This type of irregularity can be seen on every bad quality road. The sine-sweep irregularity is a longitudinal sinusoidal road distortion with growing frequency and it puts high stress on the suspension system. This irregularity represents a typical road at a bus stop, having a rolled-up structure caused by repeated braking of heavy road vehicles. The simulation route has $10 \mathrm{~cm}$ bump irregularities at $108 \mathrm{~m}$, multiple bumps at $750 \mathrm{~m}$ and $1150 \mathrm{~m}$ and sine-sweep irregularity at $1500 \mathrm{~m}$.

Fig. 7. shows the selected values of the scheduling variable based on the method described in Section 3. Constant scheduling variable is applied for the non-adaptive scenario in order to compare the two scenarios.

Vertical, longitudinal and lateral accelerations show the driving comfort performance. These results are shown in Fig. 8, while vertical, longitudinal and lateral accelerations have been improved with the proposed method. The biggest change is in the several bumps scenario/location, where the road has bad quality with discontinuities in the asphalt. The changes in $10 \mathrm{~cm}$ bumps and sine-sweep scenarios/locations are not as big as that of the several bumps road irregularities.

Suspension deflection performance, which is related to vehicle stability also has been improved with the introduced adaptive suspension system, especially at the road distortions.

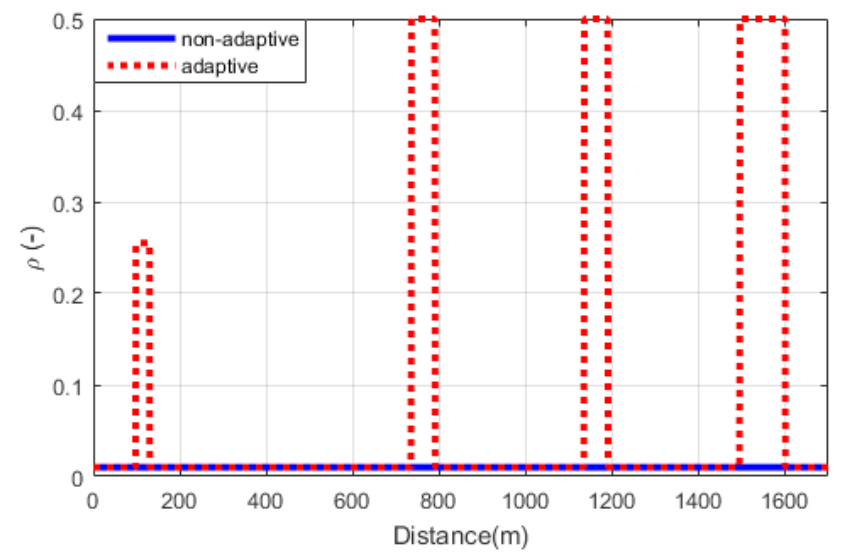

Fig. 7 Selected scheduling variable

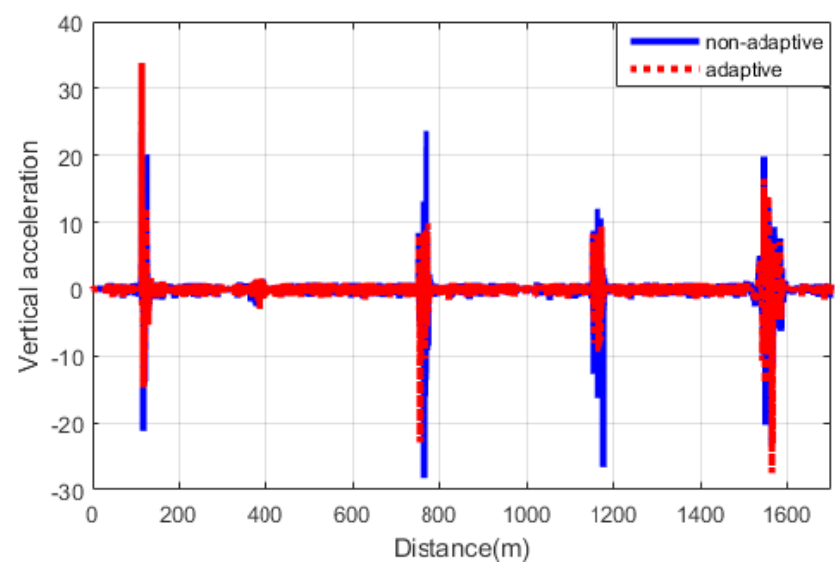

(a)

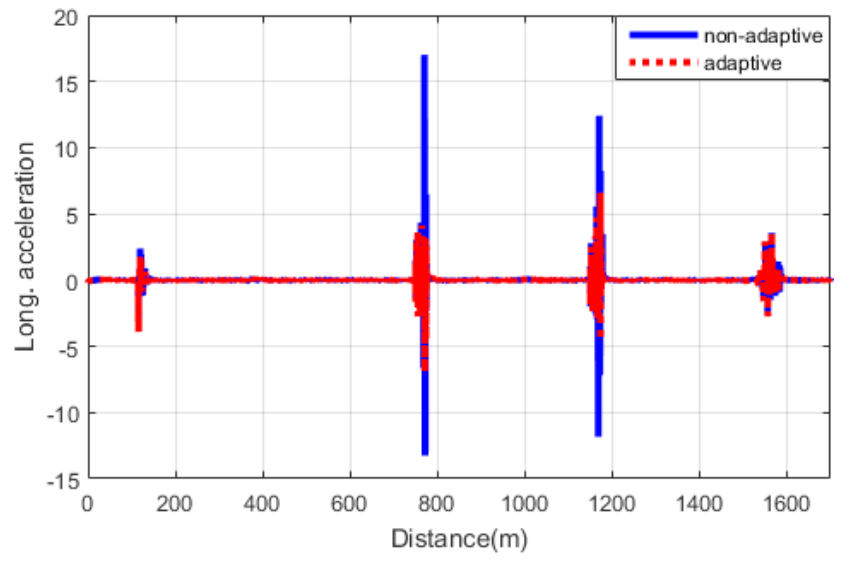

(b)

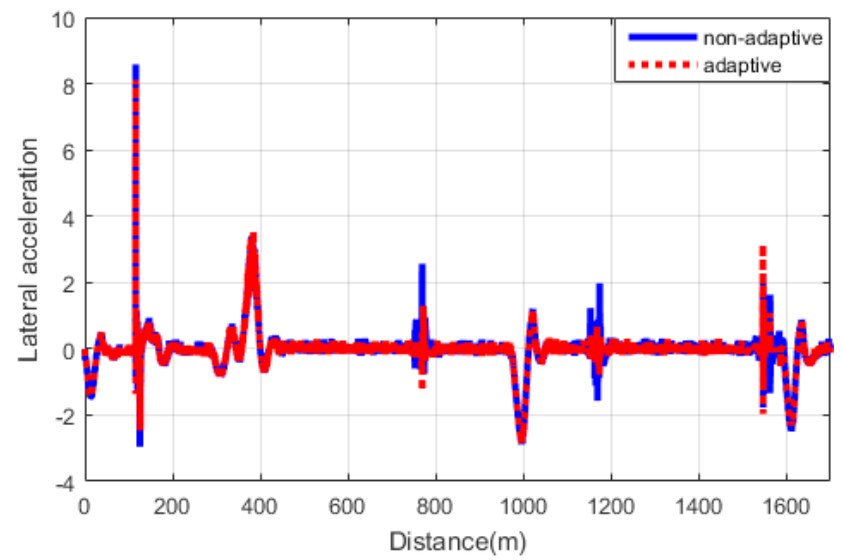

(c)

Fig. 8 (a) Vertical acceleration, (b) longitudinal acceleration, (c) lateral acceleration

Note, that at the flat road, both adaptive and conventional semi-active control have same scheduling variables, thus performances here does not differ significantly (Figs. 9 and 10).

The actuated force minimization was also considered in the design process. Fig. 11 shows these actuated forces for each suspension. It has been shown, that there are significant changes with the adaptive control method. 


\section{Conclusion}

This study introduced a road adaptive semi-active suspension control method, where a trade-off between comfort and stability is possible in order to achieve desirable performance results at different road irregularities. The effectiveness of the introduced control method has

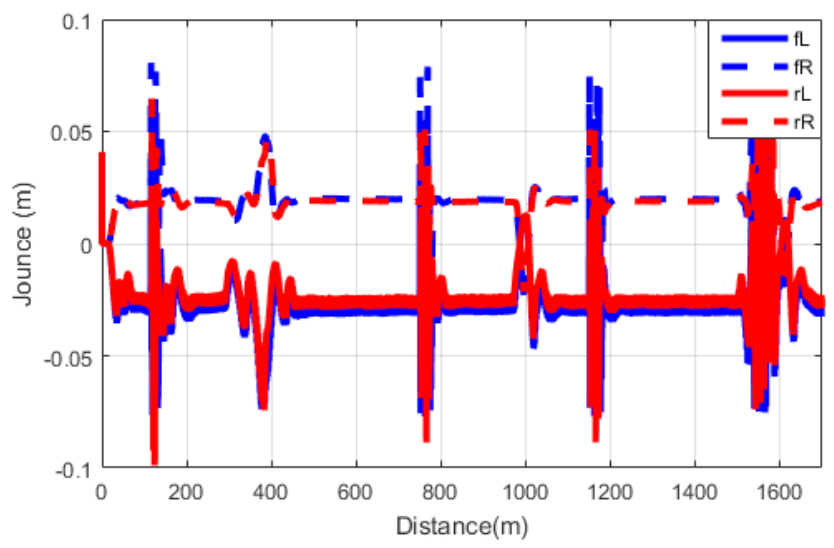

(a) been validated in TruckSim environment by comparing two different simulations, one with adaptive semi-active suspension control and another with a conventional semi-active suspension control. The results show that the performances of tire deformation, suspension deflection, and vertical acceleration have been improved.

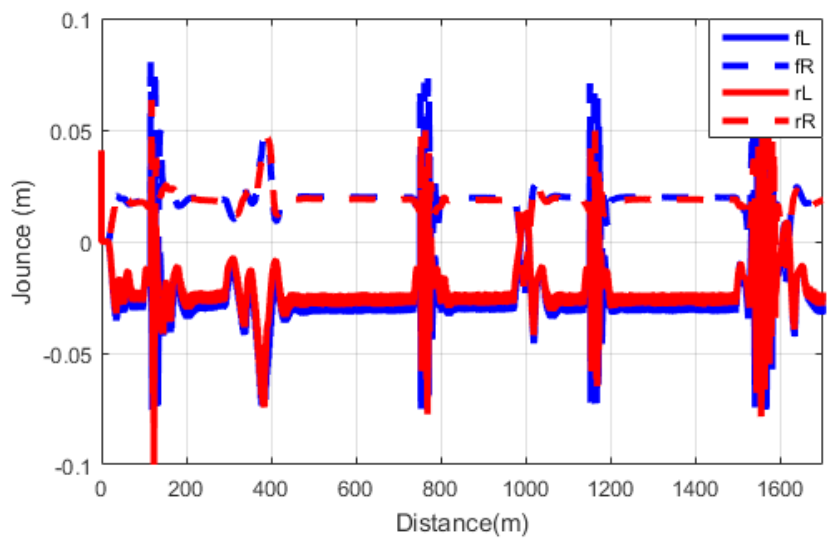

(b)

Fig. 9 Suspension deflection, (a) non-adaptive, (b) adaptive

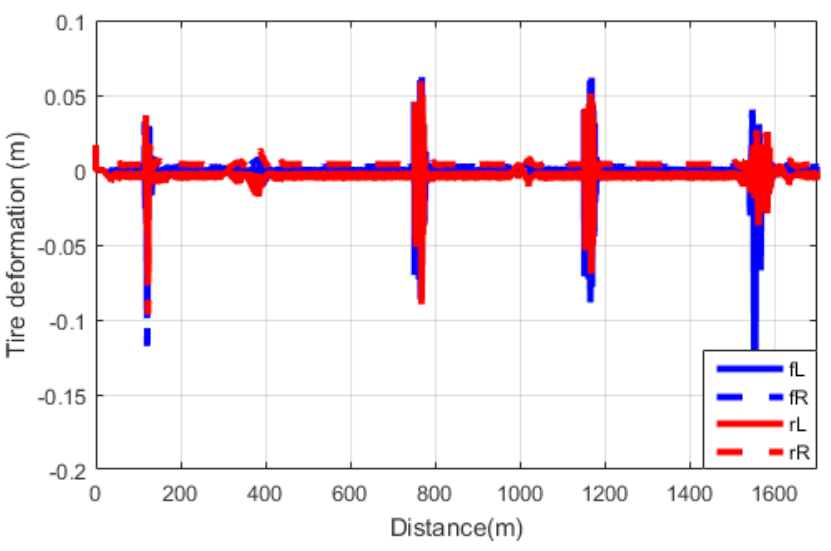

(a)

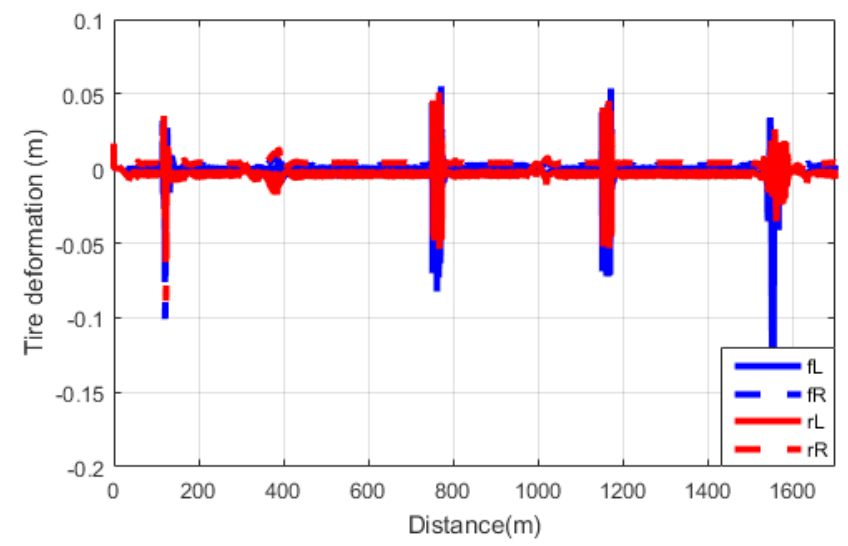

(b)

Fig. 10 Tire deformation, (a) non-adaptive, (b) adaptive

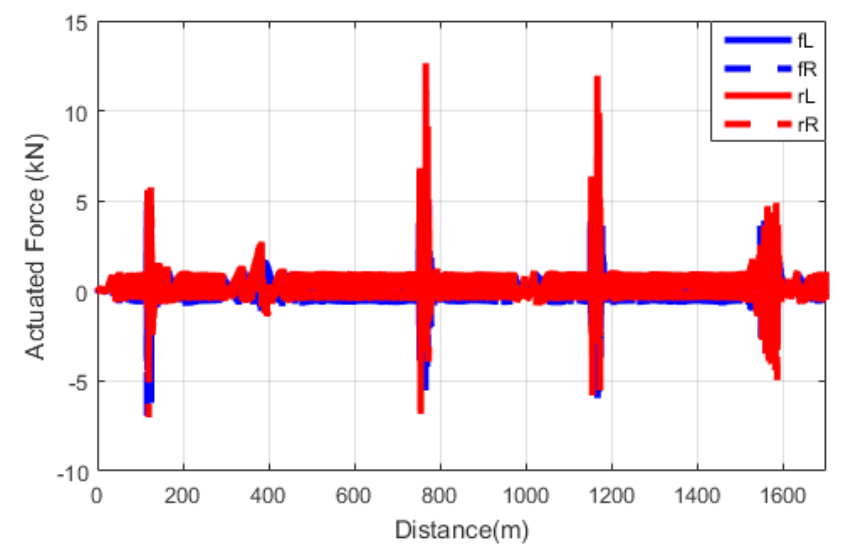

(a)

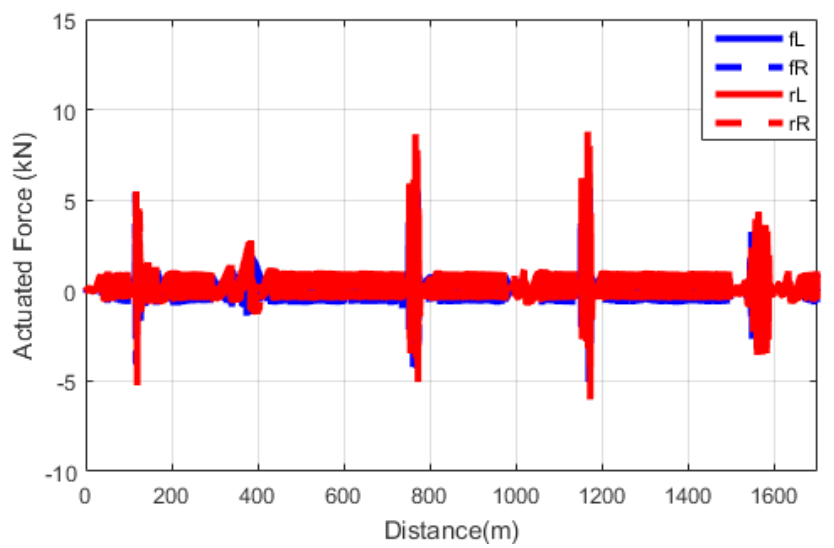

(b)

Fig. 11 Actuated forces, (a) non-adaptive, (b) adaptive 


\section{Acknowledgement}

The research presented in this paper, carried out by Institute for Computer Science and Control was supported by the Ministry for Innovation and Technology and the National Research, Development and Innovation Office within the framework of the National Lab for Autonomous Systems. This paper was partially supported by the National Research, Development and Innovation Office through

\section{References}

Basargan, H., Mihály, A., Gáspár, P., Sename, O. (2020a) "Integrated multi-criteria velocity and semi-active suspension control based on look-ahead road information", In: 28th Mediterranean Conference on Control and Automation, Saint-Raphaël, France, pp. 25-30. https://doi.org/10.1109/MED48518.2020.9182953

Basargan, H., Mihály, A., Gáspár, P., Sename, O. (2020b) "Adaptive semi-active suspension control considering look-ahead road Information and irregularities", presented at 17th Mini Conference on Vehicle System Dynamics, Identification and Anomalies, Budapest, Hungary, November 102020.

Basargan, H., Mihály, A., Gáspár, P., Sename, O. (2021) "Adaptive semi-active suspension and cruise control through LPV technique", Applied Sciences, 11(1), Article number: 290. https://doi.org/10.3390/app11010290

Bokor, J., Balas, G. (2005) "Linear parameter varying systems: A geometric theory and applications", IFAC Proceedings Volumes, 38(1), pp. 12-22.

https://doi.org/10.3182/20050703-6-CZ-1902.00003

Fleps-Dezasse, M., Ahmed, M. M., Brembeck, J., Svaricek, F. (2016) "Experimental evaluation of Linear Parameter-Varying semi-active suspension control", In: IEEE Conference on Control Applications (CCA), Buenos Aires, Argentina, pp. 77-84. https://doi.org/10.1109/CCA.2016.7587825

Gong, M., Chen, H. (2020) "Variable damping control strategy of a semi-active suspension based on the actuator motion state", Journal of Low Frequency Noise, Vibration and Active Control, 39(3), pp. 787-802.

https://doi.org/10.1177/1461348418825416

International Organization for Standardization (1997) "ISO 2631-1:1997 Mechanical vibration and shock-evaluation of human exposure to whole-body vibration-Part 1: General requirements", International Organization for Standardization, Geneva, Switzerland.

Liu, C., Chen, L., Yang, X., Zhang, X., Yang, Y. (2019) "General theory of skyhook control and its application to semi-active suspension control strategy design", IEEE Access, 7, pp. 101552-101560. https://doi.org/10.1109/ACCESS.2019.2930567

Madhavan Rathai, K. M., Sename, O., Alamir, M. (2019a) "Reachability based Model Predictive Control for Semi-active Suspension System", In: 2019 Fifth Indian Control Conference (ICC), New Delhi, India, pp. $68-73$.

https://doi.org/10.1109/INDIANCC.2019.8715601 the project "Integration of velocity and suspension control to enhance automated driving comfort in road vehicles" (NKFIH 2018-2.1.13- TÉT -FR). The research was also partially supported by the Hungarian Government and cofinanced by the European Social Fund through the project "Talent management in autonomous vehicle control technologies" (EFOP-3.6.3-VEKOP- 16-2017- 00001).

Madhavan Rathai, K. M., Alamir, M., Sename, O. (2019b) "Experimental Implementation of Model Predictive Control Scheme for Control of Semi-active Suspension System", IFAC-PapersOnLine, 52(5), pp. 261-266. https://doi.org/10.1016/j.ifacol.2019.09.042

Moaaz, A. O., Ghazaly, N. M. (2019) "Semi-active suspension system control using skyhook and groundhook controller", International Journal of Advanced Science and Technology, 28(18), pp. 424-433. [online] Available at: http://sersc.org/journals/index.php/IJAST/ article/view/2463 [Accessed: 08 December 2020]

Morato, M. M., Nguyen, M. Q., Sename, O., Dugard, L. (2019) "Design of a fast real-time LPV model predictive control system for semi-active suspension control of a full vehicle", Journal of the Franklin Institute, 356(3), pp. 1196-1224.

https://doi.org/10.1016/j.jfranklin.2018.11.016

Pham, T. P. (2020) "LPV observer and Fault-tolerant control of vehicle dynamics: application to an automotive semi-active suspension system", PhD dissertation, Université Grenoble Alpes. Available at: https://hal.univ-grenoble-alpes.fr/tel-02961042 [Accessed: 08 December 2020]

Shimoya, N., Katsuyama, E. (2019) "A study of vehicle ride comfort using triple skyhook control for semi-active suspension system", Transactions of Society of Automotive Engineers of Japan, 50(6), pp. 1631-1636. https://doi.org/10.11351/jsaeronbun.50.1631

Tseng, H. E., Hrovat, D. (2015) "State of the art survey: active and semi-active suspension control", Vehicle system dynamics, 53(7), pp. 1034-1062. https://doi.org/10.1080/00423114.2015.1037313

Wu, F., Yang, X. H., Packard, A., Becker, G. (1995) "Induced norm control for LPV system with bounded parameter variation rates", In: American Control Conference, Washington, DC, USA, pp. 2379-2383. https://doi.org/10.1109/ACC.1995.531398

Yu, S., Zhang, J., Xu, F., Chen, H. (2019) " $H_{\infty}$ Control of Semi-Active MR Damper Suspensions", In: 2019 12th Asian Control Conference, Kitakyushu, Japan, France, pp. 337-342.

Ye, H., Zheng, L. (2019) "Comparative study of semi-active suspension based on LQR control and $H_{2} / H_{\infty}$ multi-objective control", In: Chinese Automation Congress, Hangzhou, China, pp. 3901-3906. https://doi.org/10.1109/CAC48633.2019.8996771

Zhang, L., Wang, Y., Wu, G., Liu, Z. (2017) "Hybrid model predictive control of semi-active suspension with variable damping shock absorber", Journal of Xi'an Jiaotong University, 51(11), pp. 156-164. 